\title{
Advanced Physics of Thermoelectric Generators and Photovoltaic Cells
}

\author{
Salama Abdelhady \\ Faculty of Energy Engineering, Aswan University, Aswan, Egypt
}

Email address:

Salama_abdelhady@hotmail.com

\section{To cite this article:}

Salama Abdelhady. Advanced Physics of Thermoelectric Generators and Photovoltaic Cells. American Journal of Physics and Applications. Vol. 6, No. 5, 2018, pp. 133-141. doi: 10.11648/j.ajpa.20180605.14

Received: October 4, 2018; Accepted: October 22, 2018; Published: December 3, 2018

\begin{abstract}
The measured efficiencies of modern photovoltaic solar cells that exceed the limit determined by Shockley and Queisser indicate a need for advanced physics to solve such conflict. Similarly, the duality confusion represents another conflict that acquires new physics. Such conflicts and confusions were recently solved by using an innovative definition of the nature of electric current as electromagnetic waves of electric potential. This definition was used to find also plausible physical explanation of the results of Tesla's experiment of transmission of electric power in space and the success of Faraday in polarizing light by electric field in one of his experiments. Additionally, literature failed to find plausible physical explanation of estimating the electric potential of the output electric current from thermopiles and thermoelectric generators as the sum of electric potentials gained in crossing the junctions of these devices. It is shown in this paper that the introduced nature of electric current leads to advanced and plausible physical explanation of such results. It is shown also in this paper that the electric potential of the output electric current from multijunction photovoltaic cells can be estimated, similar to the thermopiles and TEG, as sum of electric potentials gained in crossing the junctions of these cells. Such similarity between the relations applied in estimating the gained potentials in all these multijunction-devices in addition to the relation found by Goldsmid and Sharp between the Seebeck coefficient and the energy bandgap prove that the Photovoltaic effect and the Seebeck effect corresponds simply to the same phenomenon. In other words; the gained potential in photovoltaic cells is generated by the thermal potential of the incident radiation and the difference of the Seebeck coefficients of the materials of its junctions. Such advanced physics may represent a gateway to understand other phenomena in the nature.
\end{abstract}

Keywords: Thermopiles, Thermoelectric Generators, Multi-Junctions Photovoltaic Cells, Seebeck Effect, Photovoltaic Effect, Energy Bandgap

\section{Introduction}

Through an entropy approach in previous studies, the Maxwell's wave equations were casted into an energy frame of reference by replacing the time in these equations by entropy as follows [1-3].

$$
\begin{aligned}
& \left(\nabla^{2}-\frac{1}{c^{2}} \frac{\partial^{2}}{s^{2}}\right) \mathrm{E}=0 \\
& \left(\nabla^{2}-\frac{1}{c^{2}} \frac{\partial^{2}}{s^{2}}\right) \mathrm{H}=0
\end{aligned}
$$

where $\mathrm{c}$ is the speed of light, $\mathrm{E}$ is the electric field and $\mathrm{H}$ is the magnetic field. Equations (1) and (2), succeeded in visualizing an innovative definition of electric current as electromagnetic waves of electric potential in a frame of energy coordinates defined by the Electric Field, E, as the vertical axis, the magnetic field, $\mathrm{H}$, as the abscissa and the entropy, $\mathrm{s}$, as the ordinate, as shown in Figure $1[4,5]$.

Additionally, it was possible by such modified Maxwell's equations to find an analytical model of the introduced nature of electric current as one of its solutions which has initial electric potential of " $+/-\Delta \bar{E}$ " as follows [6]:

$$
\begin{gathered}
E(r, s)=E \cos (k r+\omega s+\varphi)+/-\Delta \overline{\mathrm{E}} \\
H(r, s)=H \cos (k r+\omega s+\varphi)
\end{gathered}
$$

where $r$ is the axial propagation of the wave, $\omega$ is the frequency of the wave and $\varphi$ is the wave phase shift. Such equations indicate a solution for the success of Faraday in adding electric potential, or electrifying, the light, as 
electromagnetic waves, by electric field and converting it into electric current in one of his experiments [7]. A graphical presentation of (3) and (4) in the E-H-S diagram is shown in Figure 2 [6], where the E-axis of the electric oscillations of the waves is shifted by $\Delta \bar{E}$. Figure 2 represents in general the flow of electric current as a flow of electromagnetic waves of electric potential $\Delta \overline{\mathrm{E}}$, which maybe positive (as a charge of a proton) or negative (as a charge of an electron) [6].

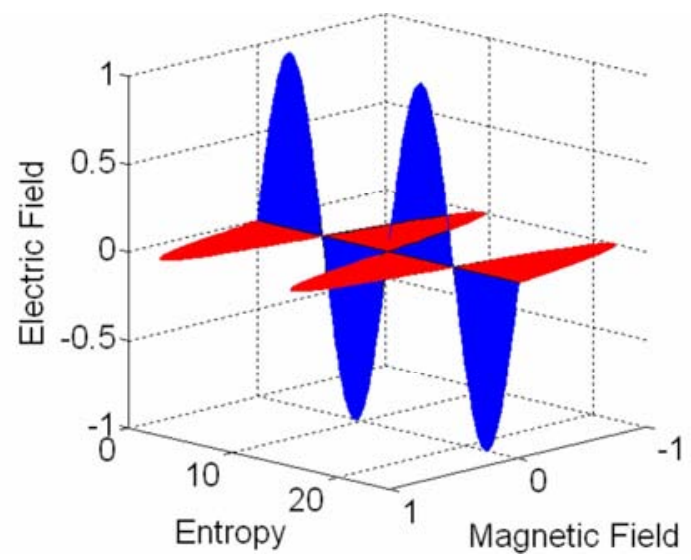

Figure 1. Representation of flow of Electromagnetic waves in Energy frame of references composed of the energy coordinates: Electric field, Magnetic field, and Entropy, E-H-s coordinates, where the flow of the electric wave is shown in the E-s plane and the flow of the magnetic wave is shown in the $\mathrm{H}$ s plane [1, 2].

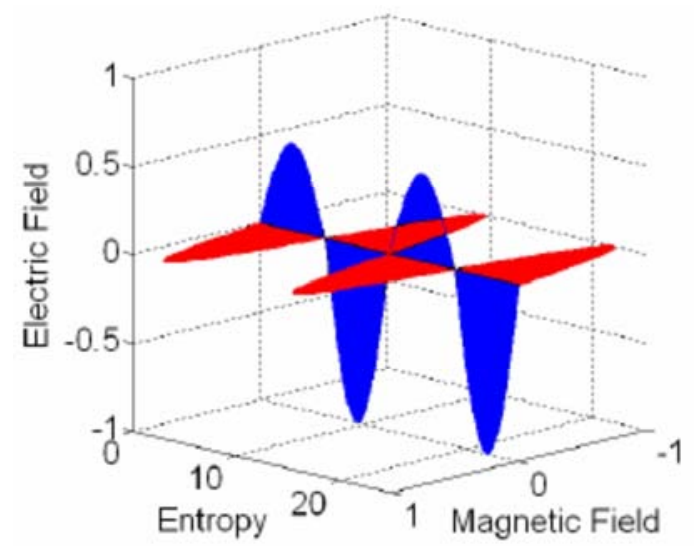

Figure 2. Flow of negative electric charges as flow of electromagnetic waves of "-ve" potential where electric energy in the E-s plane is oscillating around the negative potential " $-\Delta \bar{E}$ " $[1,2]$.

The Tesla's experiment of transmission of electric power in space as electromagnetic waves of extremely high potential represents also a proof of such representation of electric current [8]. Additionally, the lightening phenomenal of discharging electric current as electromagnetic waves of extremely high electric potential that shocks anything in its way proves this nature of electric current [9]. It is found in literature that the measured electric potential of the output electric current from thermopiles and thermoelectric generators is estimated as the sum of electric potentials gained in crossing the junctions of these devices. Such results lack the plausible physical explanation. Similarly; the measured efficiencies of the advanced photovoltaic cells exceed the limit found by classical physics of such devices, as found by Shockley and Queisser [10]. Relying on the introduced innovative nature of electric current in this study, we shall develop the classical physics of the thermopiles, thermoelectric generators or photovoltaic cells to find plausible physical explanation of the found measurement results for thermopiles, thermoelectric generators and multijunction photovoltaic cells. The derived advanced physics will involve the following relation developed by Goldsmid and Sharp [11], to find a relation between the Seebeck effect and the photovoltaic effect in such applications:

$$
E_{g}=2 \text { e }\left|\alpha_{\max }\right| T_{\max }
$$

Where $E_{g}$ denotes the energy bandgap of the material of semiconductor, $e$ is the elementary charge, $\alpha_{\text {max }}$ represents the maximum Seebeck coefficient of the material, and $T_{\max }$ is the temperature of material at which the material attains its maximum value of Seebeck coefficient, i.e. $\alpha_{\max }$.

\section{Advanced Physics of Thermocouples and Thermopiles}

The thermocouple consists of two wires of different metals connected at two points, i.e. two junctions, such that a potential difference is developed between the two junctions in proportion to the temperature difference between the junctions. The thermoelectric effect in a thermocouple is defined as the production of an electromotive force or potential difference, and consequently an electric current in a thermocouple. Considering an ideal thermocouple, the open circuit voltage $V$ obtained is proportional to the temperature difference $\Delta T$ between the junctions constructed of conductors $\mathrm{A}$ and $\mathrm{B}$ as follows $[12,13]$ :

$$
\Delta V=\propto_{A B} \Delta T
$$

Where " $\propto_{A B}$ " is the relative Seebeck coefficient, expressed in $\mu V / K$. This coefficient depends not only on the temperature, but also on the choice of the two materials used in the thermocouple. The magnitude of the relative Seebeck coefficient, $\alpha_{A B}$ of the junction of two materials or metals $A$ and $\mathrm{B}$ can be evaluated as the difference between the Seebeck coefficients of each metal as follows $[14,15]$ :

$$
\propto_{A B}=\propto_{B}-\propto_{A}
$$

The direct relation between the produced electric potential and the thermal potential of the flowing energy plays the main role in the use of thermocouples in temperature measurements. In photoelectric literature, the difference in the energy bandgaps of the PN junction's materials is considered as the mechanism of creating the potential difference across the junction [16]. As the Seebeck effect is characterized by the difference between the Seebeck coefficients of the materials of the thermocouple junction, and the photoelectric effect is characterized by the difference 
in the band gap of the materials of the photovoltaic cell. Hence, the relation of Goldsman and Sharp between the Seebeck coefficient and the energy bandgap of materials, So, equation (5) signifies a relation between the Seebeck effect and the photovoltaic effect. In other words, it is possible to refer to the photovoltaic effect as a result of the difference between the Seebeck coefficients of the materials of the junction of the photovoltaic cell. Finally it can be concluded the equivalence of the Seebeck effect and the Photovoltaic effect. So, defining the electric current as flow of electromagnetic waves of electrical potential, it is possible to consider the crossing of junctions of materials of different Seebeck coefficients, or of different energy bandgaps as the mechanism that replaces the thermal potential of the flowing heat by electrical potential and, so, ionizes the crossing photons. Consequently, thermopiles are used, i.e. several junction pairs are connected in series as shown in Figure 3 [17], in order to increase the developed output voltage.

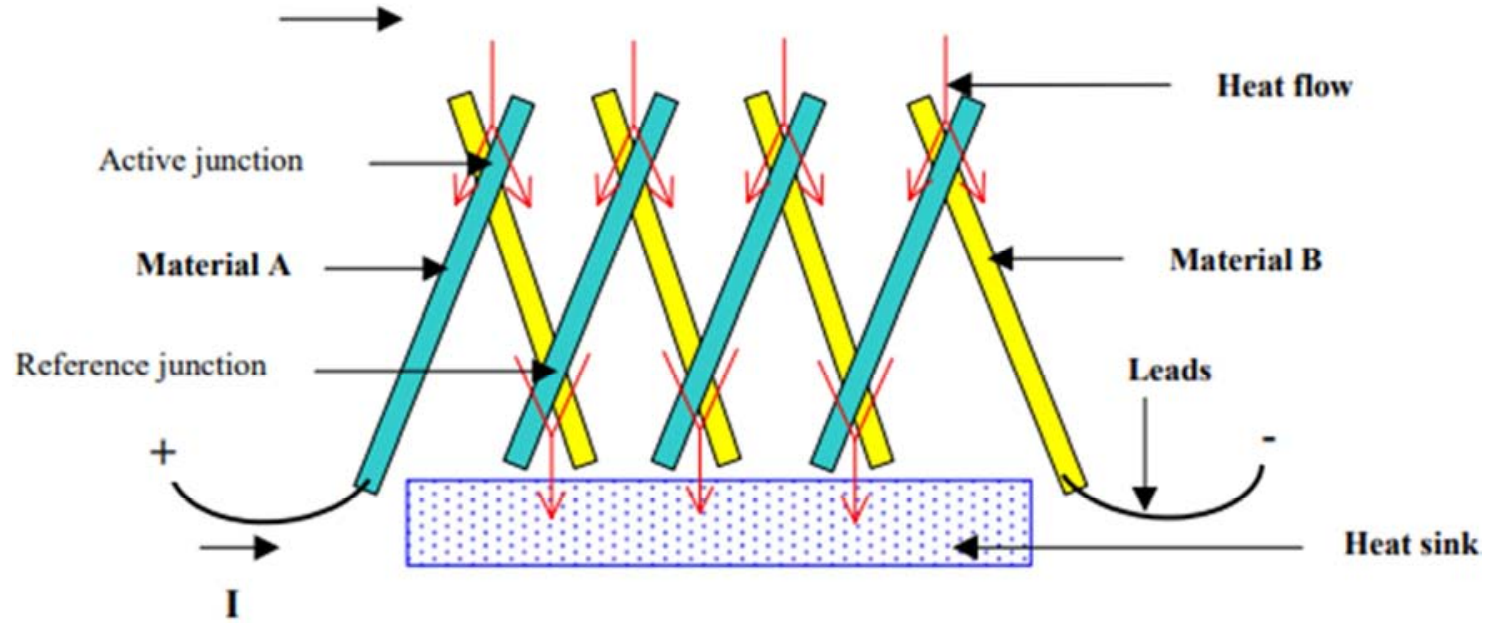

Figure 3. A thermopile: composed of thermocouple junction-pairs of two metals connected electrically in series to increase the generated E. M. F [17].

As the thermocouple junction pairs are placed in series as shown in Figure 3; then the output voltage can be estimated as the sum of the gained electric potentials during the flow of the electromagnetic waves of thermal potentials in a unique direction across the successive junctions by the Seebeck effect. So, it is possible to estimate the total gain as the sum of these individual gains at successive junctions as follows:

$$
\begin{gathered}
\Delta V=\propto_{A B}\left(T_{h}-T_{l}\right)+\propto_{B A}\left(T_{l}-T_{h}\right)+\propto_{A B}\left(T_{h}-\right. \\
\left.T_{l}\right)+\propto_{B A}\left(T_{l}-T_{h}\right)+\propto_{A B}\left(T_{h}-T_{l}\right)+\cdots
\end{gathered}
$$

According to Eq. (7); $\propto_{A B}=\propto_{B}-\propto_{A}$,

Then,

$$
\propto_{B A}=\propto_{A}-\propto_{B}=-\propto_{B A}
$$

And as;

$$
\left(T_{h}-T_{l}\right)=-\left(T_{l}-T_{h}\right)
$$

By using Eqs. (9) and (10) to replace the corresponding terms in Eq. (8), then the total electric potential gained by the flowing electromagnetic waves by Seebeck effect is as follows:

$$
\Delta V=\sum\left[\propto_{A B}\left(T_{h}-T_{l}\right)\right]=n \propto_{A B}\left(T_{h}-T_{l}\right)
$$

Equation (11) indicates that the electric potential of a thermopile is duplicated by the number of the used junctions, i.e. n. Such equation fits the measured electric potential of the output current from thermopiles. However, these results cannot have a plausible physical explanation if the electric current was defined as a flow of electrons $[18,19]$. So, the definition of electric current as a flow of electromagnetic waves of gained electric potential during crossing the successive junctions of the thermopile offers a plausible physical explanation that fits the found measurement results as expressed by (11) [20].

According to the relation (5) between the Seebeck coefficient and the energy bandgap as found by Goldsmid and Sharp [11], the electric potential gained by electromagnetic waves (heat) when crossing the junctions of a thermopile, which depends on the difference of the Seebeck coefficients, bears also a dependence on the difference between their energy bandgaps. This conclusion proves also a relation between the thermoelectric effect (Seebeck effect) and the Photovoltaic effect. In other words, we may state that the Seebeck effect, defined by the Seebeck coefficients of the materials of a junction is a similitude of the photovoltaic effect that is characterized by the difference in the energy bandgaps.

\section{Advanced Physics of Thermoelectric Generators}

A thermoelectric generator (TEG) is defined in literature as a Seebeck generator or a solid state device that converts heat flow of thermal potential directly into electrical energy by the Seebeck effect [20. 21]. However; the Seebeck effect as a thermoelectric effect cannot explain the conversion of heat into electric current if the heat flow is defined as flow of electromagnetic waves of thermal potential and the electric current is defined as flow of electrons. A plausible physical 
explanation of this definition of TEG can be accepted if we define the electric current as electromagnetic waves which gains accumulated electric potentials during its flow through the successive junctions of the generator.

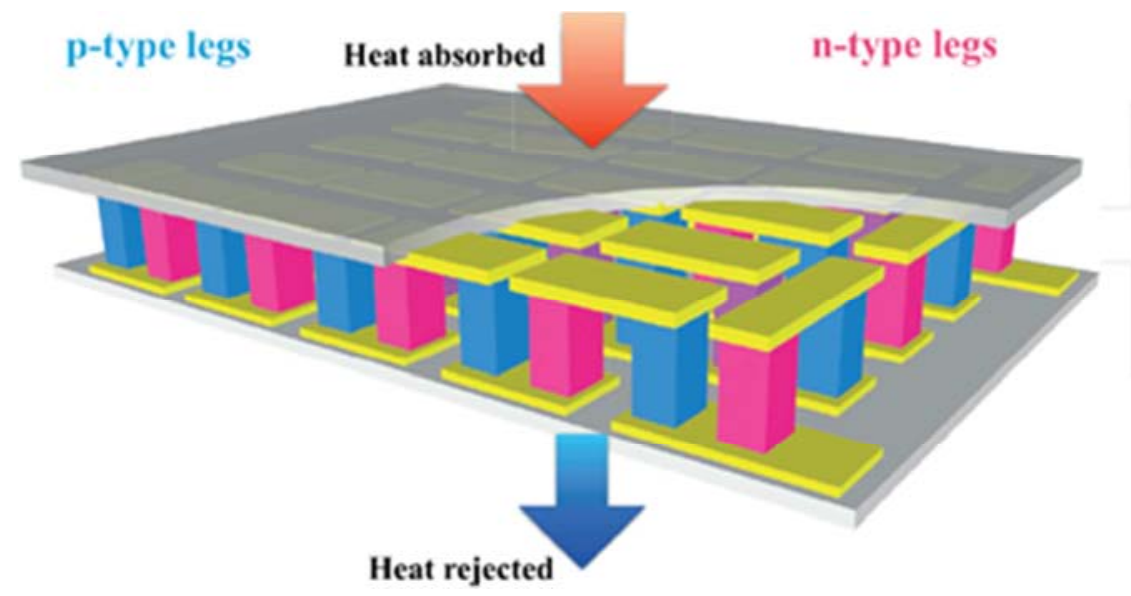

Figure 4. Schematic diagram of a thermoelectric generation (TEG) module: Composed of p-type and n-type legs that are bonded together in series to increase the electric potential of the output current [21].

A classical TEG is shown in Figure 4. The shown thermoelectric module consists of units, or legs, of $n$ and $p$ type semiconducting materials connected electrically in series and thermally in parallel. Figure 4 shows a module of the TEG that is similar in principles to the operation of the thermopile shown in Figure 3 [21]. As the junction pairs in this module are connected in series; then the output voltage can be estimated as the sum of the gained electric potentials during the flow of the electromagnetic waves of thermal potentials in a unique direction across the successive junctions by the Seebeck effect. So, considering the flow of electric current as flow of electromagnetic waves of accumulating potentials, it is possible to estimate the total gain in the electric potential as the sum of these individual gains at successive junctions as follows.

$$
\begin{gathered}
\Delta V=\propto_{p n}\left(T_{h}-T_{l}\right)+\propto_{n p}\left(T_{l}-T_{h}\right)+\propto_{p n}\left(T_{h}-T_{l}\right)+ \\
\propto_{n p}\left(T_{l}-T_{h}\right)+\propto_{p n}\left(T_{h}-T_{l}\right)+\propto_{n p}\left(T_{l}-T_{h}\right)
\end{gathered}
$$

However;

According to Eq. (7): $\propto_{p n}=\propto_{p}-\propto_{n}$

Then,

$$
\propto_{n p}=\propto_{n}-\propto_{p}=-\propto_{p n}
$$

While,

$$
\left(T_{h}-T_{l}\right)=-\left(T_{l}-T_{h}\right)
$$

Substituting (13) and (14) in (12), the total electric potential gained by the flowing electromagnetic waves by Seebeck effect can be calculated as follows [22]:

$$
\Delta V=\sum\left[\propto_{p n}\left(T_{h}-T_{l}\right)\right]=n \propto_{p n}\left(T_{h}-T_{l}\right)
$$

Such result shows the output potential of the generator increases by the number of junctions "n," the Seebeck coefficient of the junctions " $\left|\propto_{p n}\right|$," and the temperature difference between the plates of the generator " $\left(T_{h}-T_{l}\right)$ ". However, the relation (15) is used in literature for calculating the potential of the output of TEG without physical proof or explanation. Such equation shows that the flowing electromagnetic waves, or heat, gain electric potentials by crossing the successive junctions of the thermoelectric generator and is converted into electric current of accumulated electric potentials [23]. The similarity of (11) for thermopiles and (15) for thermoelectric generators shows that the Seebeck effect, as a thermoelectric effect, can be explained also in case of thermoelectric generators to boost the electric potential in the flowing waves by the difference in the energy bandgaps of the crossed junctions. This conclusion is based on the definition of the electric current as a flow of electromagnetic waves of electric potential and the relation between the Seebeck effect and the Energy bandgap as found by Goldsman and sharp [11]. Accepting the relation (15), as found in literature, for estimating the electric potential of TEG imply the acceptance of the previously introduced definitions of electric current and Seebeck effect as concluded in introducing advanced physics of thermocouples and of thermopiles.

\section{Advanced Physics of Photovoltaic Cells}

Solar cells are semiconductor devices which are known to convert solar energy into electricity by the photovoltaic effect. The PV effect is traditionally defined in literature of physics as bouncing electrons by the incident photons of light across the cell's junction [24, 25]. Such definition violates the principle of conservation of momentum where the momentum of any incident photon is negligible when compared to the momentum of any orbiting electron in an atom [26]. Additionally, such explanation cannot be applied in multi-junction thin films as such electrons cannot jump freely from one junction to the adjacent junction [27, 28]. According to literature; the gained potential in the PV cells depends on the difference in the energy bandgaps of the junction's materials, i.e. the $\mathrm{p}$ and $\mathrm{n}$ junctions [24]. However, 
the relation (5) ties the energy bandgap to the Seebeck coefficient of materials as found by Goldsmid and Sharp
[11]. As previously discussed, such relation indicates the dependence of the photovoltaic effect on the Seebeck effect.

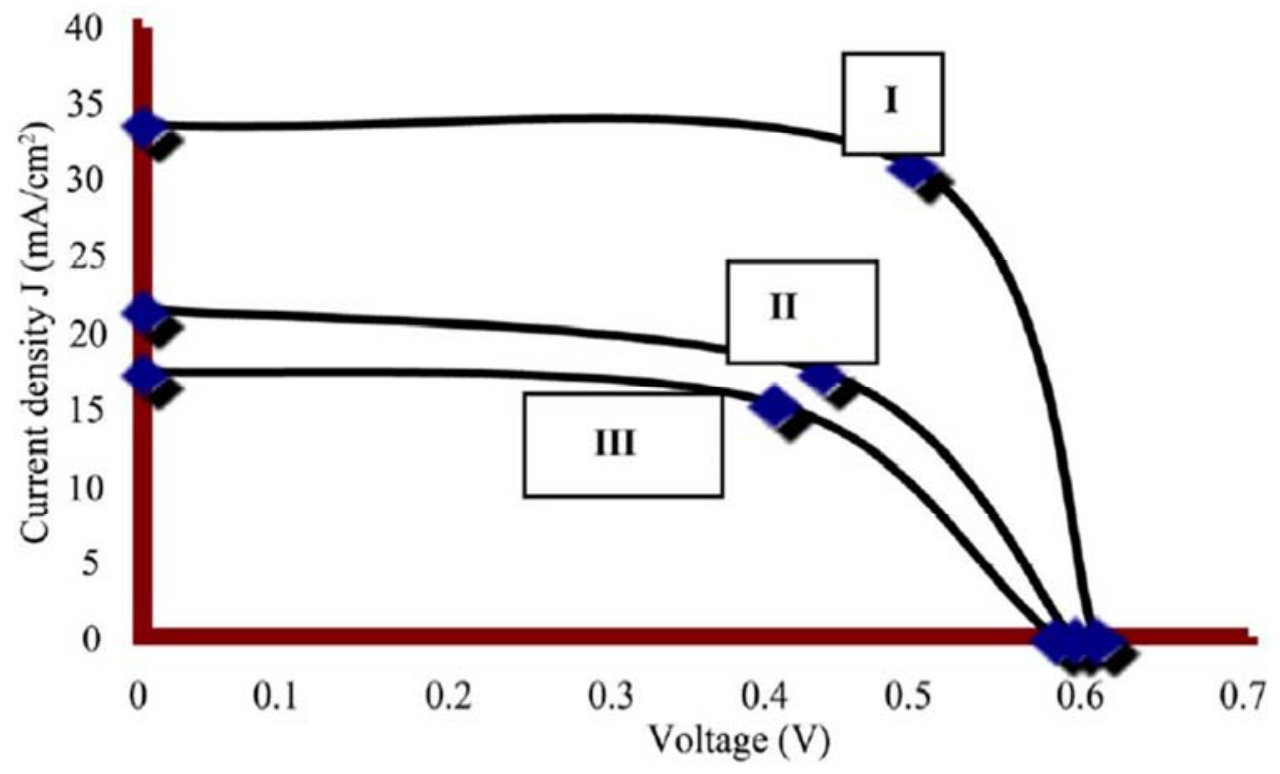

Figure 5. Typical I-V Characteritics of an illuminated single crystal silicon solar cell: operating at different values of solar radiation $100 \mathrm{mW/cm}{ }^{2}$ (I), 60 $\mathrm{mW} / \mathrm{cm}^{2}$ (II) and $40 \mathrm{~mW} / \mathrm{cm}^{2}$ (III) respectively. The open voltage potential is affected only by the temperature of the source of radiation, the sun, while the intensity of solar radiation influences the current density [24].

The results in Figure 5 shows direct the dependence of the measured open voltage potential of output current from the PV cell on the thermal potential of the incident radiation. i.e. on the temperature of the sun [29]. Such potential, indicated as " $V_{o c}$ ", is found from the figure in the range $0.6-0.7$ Volts. This gained potential corresponds to the temperature difference between the source of radiation, or the temperature of the sun, and the junction's temperature, i.e. on $T_{\text {SOURCE }}-$ $T_{\text {JUNCTION }}$. which equals in this case $5800 \mathrm{~K}$. By such measurement, the potential increase of the output electric current per unit thermal potential of the incident radiation is estimated as the quotient of the output potential and the temperature difference in the range $120-150 \mu \mathrm{V} / \mathrm{K}$. Comparing such value to the value of the estimated Seebeck coefficient of the materials of the used PV junction, both values are in the same range [30. 31, 32]. Such comparison may prove the previously concluded similarity between the PV effect and the photoelectric effect according to the relation found by Goldsmid and Sharp and approving the introduced nature of electric current as electromagnetic waves of electric potential So, the PV effect can be defined also as conversion of the incident thermal radiation on the PV cell into electric current when crossing the cell's junction by gaining electric potential due to the difference in the Seebeck coefficients, or energy bandgaps, of the junction's materials. In other words, the incident solar radiation on the PV cell will gain an electric potential which can be found by an equation similar to (6) that counts its potential according the following equation $[31,32,34]$.

$$
\Delta V=\propto_{p n}\left(T_{s}-T_{c}\right)
$$

Where; $\propto_{p n}=\propto_{p}-\propto_{n}$

$T_{s}:$ is the solar temperature,
$T_{c}:$ is the cell's temperature,

$\propto_{p}$ : Seebeck coefficient of the $\mathrm{p}$ junction,

$\propto_{n}$ : Seebeck coefficient of the $\mathrm{n}$ junction.

So, the PV cell will respond thermodynamically to the solar temperature as the heat source of the incident waves and to the cell's temperature as the heat sink. According to the Second law of thermodynamics; the maximum efficiency of this cell is limited by the Carnot-Cycle's efficiency working between these two temperatures that can be evaluated as follows [35].

$$
\eta_{\max }=1-\frac{T_{l}}{T_{h}}=1-\frac{298}{6100}=95 \%
$$

According to the previously introduced definition of Photovoltaic cells, Eq.(17) shows that the efficiency of the photovoltaic cells may reach $95 \%$ and it is not restricted to the Sckockley-Queisser limit, which was based on the Einstien's definition of photoelectric effect and the definition of electric current as flow of electrons [10]. So, such innovative definitions of photovoltaic effect and lectric current deletes the conflicts of measuring efficiencies of photovoltaic cells that exceeds the Sckockley-Queisser limit.

Multi-junction photovoltaic (MJPV) cells are used for achieving ultra-high power conversion efficiencies for both space based and terrestrial concentrator applications [36]. The technology of multijunctions PV cells is similar to the previously discussed technology of thermopiles and thermoelectric generators. According to the previously found coincidence of the photovoltaic effect and the Seebeck effect in case of single junction solar cell and the similarity of the multijunction technologies in MJPV, TEG, and thermopiles, it is possible to prove the conversion of the thermal potential into electric potential in Multijunction photovoltaic cells also 
to be actuated by the difference of the Seebeck coefficients of the materials of the junctions of the successive cells. Then, it will be possible to show the gained potential of the output current in case of MJPV cells can be evaluated by a similar relation as (11) and (15). Such statement will be discussed on the basis of available measurement data in literature as found in Figures. 6-8 [37, 38, 39, 40].

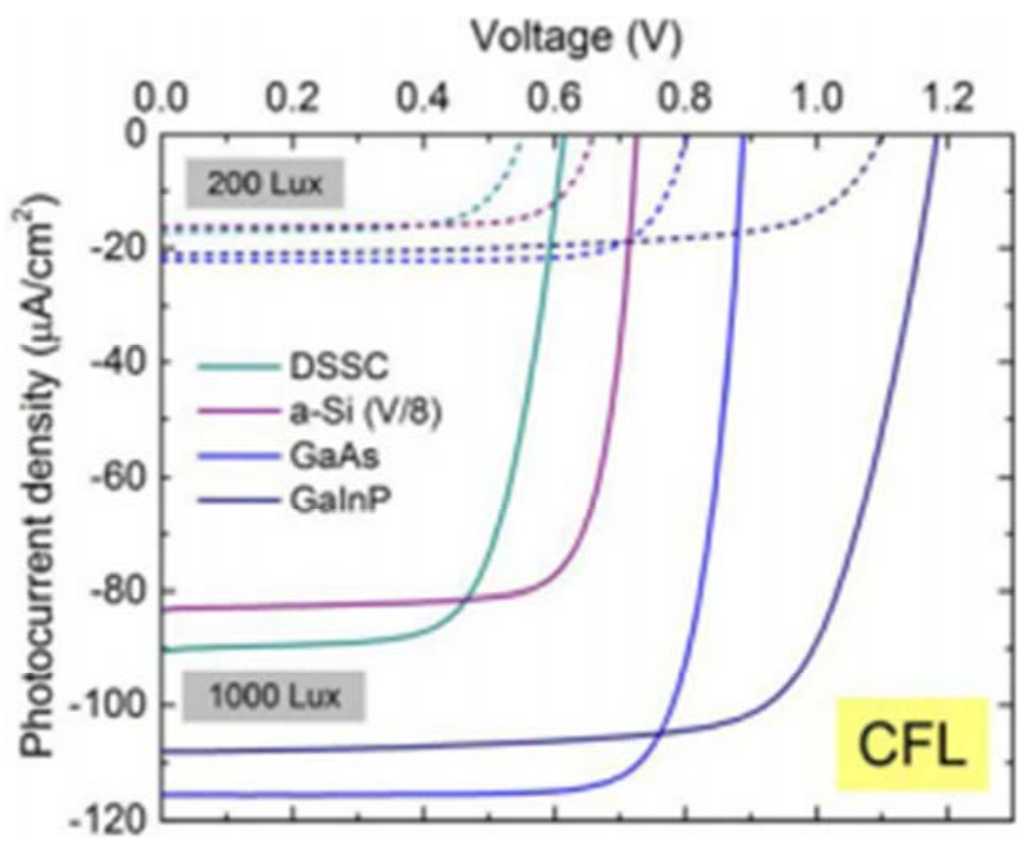

Figure 6. Measured I-V characteristics of single junction solar cells, of different semiconductor materials: DSSC, a-Si, GaAS, and GaInP each cell is operating under two luminosities 200 and 1000 lx [37].

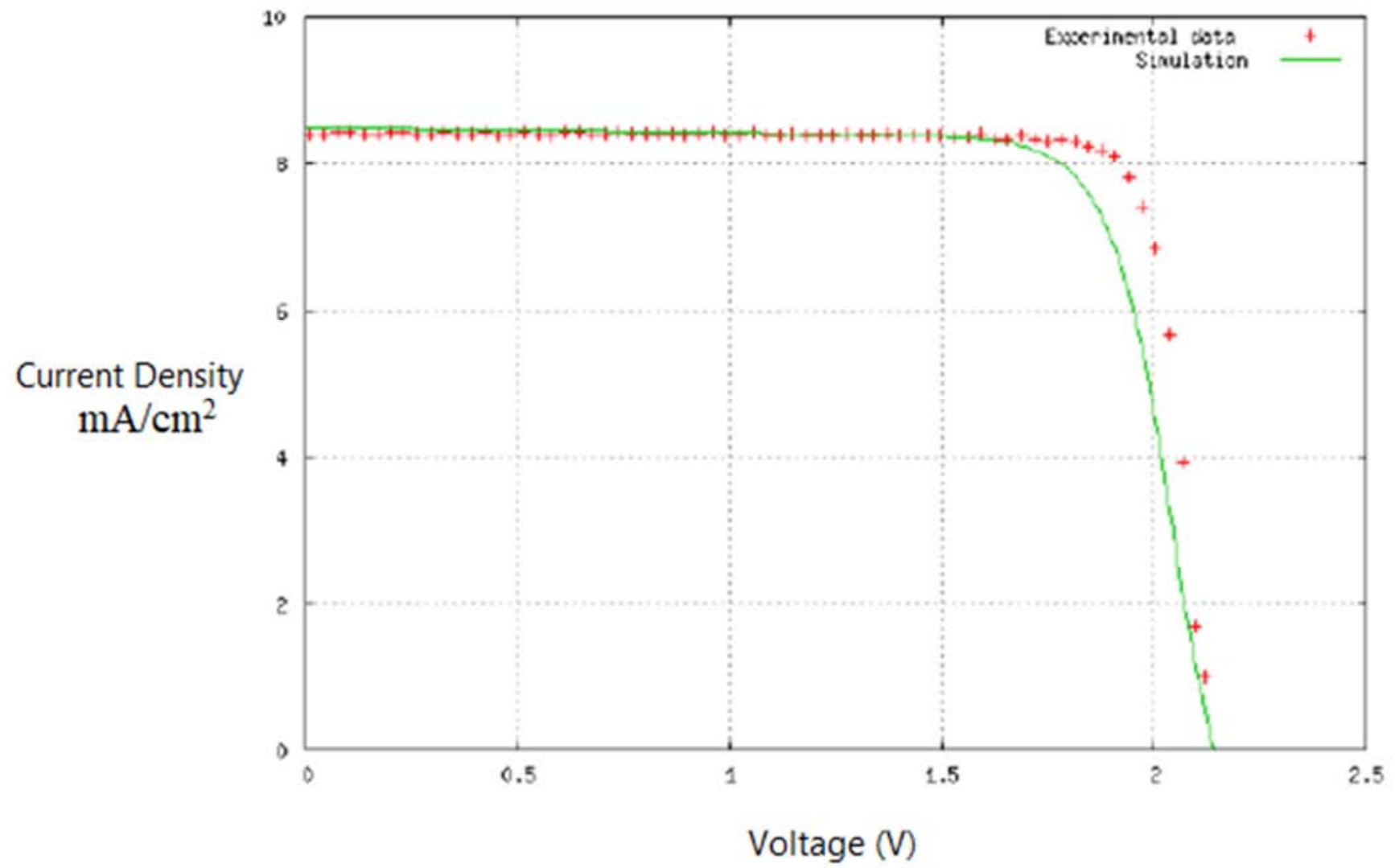

Figure 7. The measured and calculated (I-V) characteristics of dual, GaInP, GaAs, solar cells; Comparison of the calculated and measured characteristics composed of two successive GaInP, GaAs cells [38, 39]. 

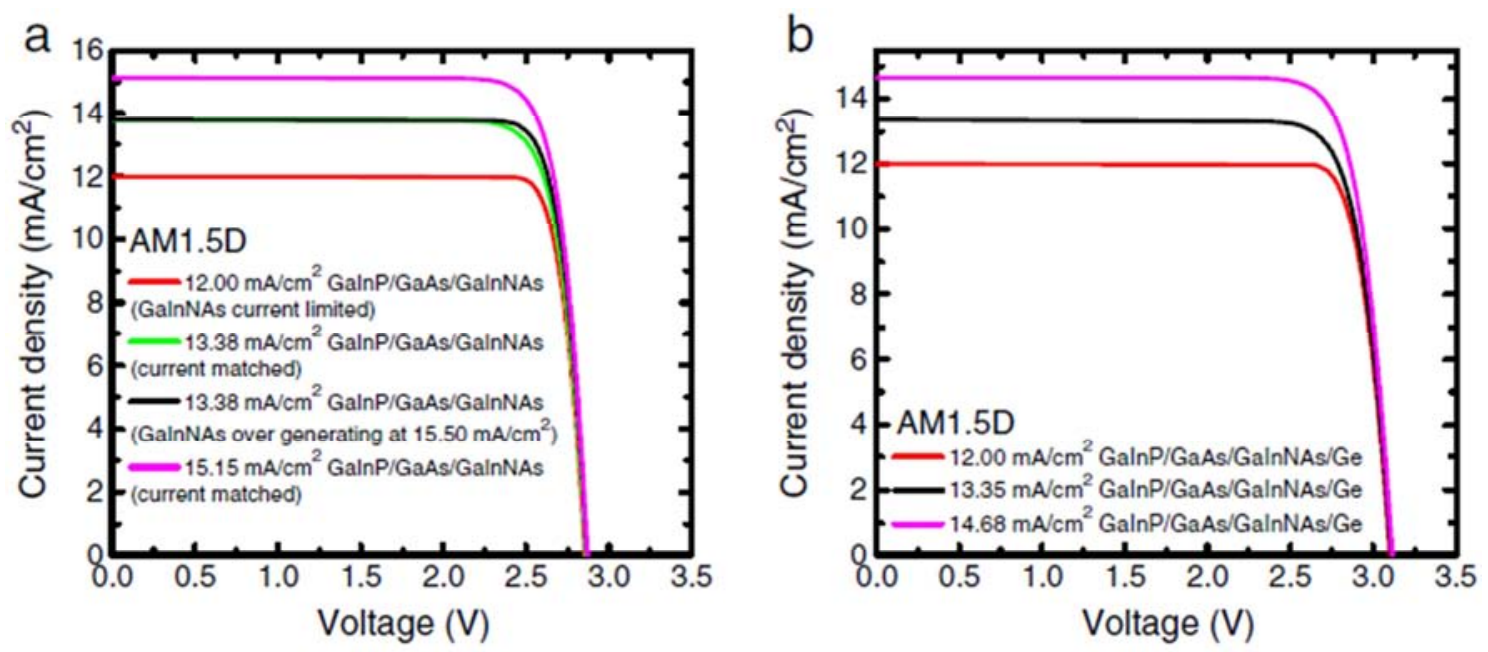

Figure 8. (a) I-V performance for MJPV cells: composed of three junctions: GaInP/GaAs/GaInNAs.(b) I-V performance for MJPV cells: composed of four junctions GaInP/GaAs/GaInNAs/Ge [40].

Figure 6 shows the I-V performance of single junction solar cells of different semi-conductors [37]. According to the seen measurement data for the GaInp and GaAs solar cells in the figure, we may calculate the potential rise per degree of the temperature of the source of incident radiation when crossing the junction of these cells as follows:

$$
\begin{aligned}
& \left(\frac{\Delta V}{T_{s}-T_{c}}\right)_{\text {GaInp }}=\frac{0.9}{5800}=155 \mu \mathrm{V} / \mathrm{K} \\
& \left(\frac{\Delta V}{T_{s}-T_{c}}\right)_{\text {GaAs }}=\frac{1.2}{5800}=206 \mu \mathrm{V} / \mathrm{K}
\end{aligned}
$$

Such values of temperature coefficients are found comparable to the Seebeck coefficients of GaInp and GaAs estimated from literature [38]. Figure 7 shows the performance of a dual junction solar cell formed of the junctions GaInp and GaAs [39]. As the whole cell receives radiation from the same source, it detects the same solar temperature $T_{h}=6100 \mathrm{~K}$. Similarly, the whole cell is subjected also the same surrounding of temperature: $T_{l}=298$ $\mathrm{K}$ which is also detected by its layers. According to the previous analysis of the single junction solar cell, the incident radiation will gain an electric potential when crossing each junction which is proportional to the same thermal potential " $T_{h}-T_{l}$." So, it is possible to calculate resultant temperature coefficient of such dual cell as the quotient of the total gained electric potential, found from Figure 7 as $2.1 \mu \mathrm{V}$, and the temperature difference $5800 \mathrm{~K}$ as follows:

$$
\left(\frac{\Delta V}{T_{S}-T_{c}}\right)_{\text {GaInp }+ \text { GaAS }}=\frac{2.1}{5800}=3.61 \mu \mathrm{V} / \mathrm{K}
$$

Comparing such result to the sum of the temperature coefficients of the single junctions GaInp and GaAs solar cells, we find that both are equal, i.e.

$$
\left(\frac{\Delta V}{T_{S}-T_{C}}\right)_{\mathrm{GaInp}+\mathrm{GaAS}}=\left(\frac{\Delta V}{T_{S}-T_{c}}\right)_{\mathrm{GaInp}}+\left(\frac{\Delta V}{T_{S}-T_{C}}\right)_{\mathrm{GaAs}}
$$

Similarity of Eq. (18) to Eq. (11) and (15), and equality of the temperature coefficients of the GaInp and GaAs photovoltaic cell to their Seebeck coefficients lead to consider the accumulation of the electric potential in the MJPV cells is due similar phenomena that are found in thermopiles and TEG and to interpret the relation (18) as follows:

$$
\propto_{\mathrm{GaInP}+\mathrm{GaAs}}==\left(\propto_{\mathrm{GaInP}}+\propto_{\mathrm{GaAs}}\right)
$$

These measurement results represent a sufficient proof of similarity of the Photovoltaic effect and the Seebeck effect. So, According to such measurements for the MJPV cells, it is possible to write for the MJPV cells a similar relation to (11) for thermopiles and to (15) for TEG as follows:

$$
\Delta V=\propto_{\text {GaInP+GaAs }}\left(T_{s}-T_{c}\right)=\left(\propto_{\text {GaInP }}+\propto_{\text {GaAs }}\right)\left(T_{s}-T_{c}\right)
$$

Such discovery of the Seebeck effect as the motivating effect in photovoltaic cells represent a logical result of the relation between the Seebeck coefficient and energy bandgap found by Goldsmid and Sharp and the definition of electric current as electromagnetic waves of accumulating electric potentials..

Figure 8 shows the I-V performance of triple junction solar cell consisting of successive layers of GaInP, GaAs and GaInNAs and the performance of four junctions - solar cell consisting of successive layers of GaInP, GanAs and GaInNAs and Ge stacked on top of each other [40]. It is possible to show that such measurements for the triple junction solar cell and the four junctions - solar in Figure 8 the validity of the equality (19) for calculating the accumulated potentials as follows:

$$
\begin{array}{r}
(\Delta V)_{3} \text { junctions }=\propto_{\mathrm{GaInP}+\mathrm{GaAs}+\mathrm{GaInNA}}\left(T_{s}-T_{c}\right)=\left(\propto_{\mathrm{GaInP}}+\propto_{\mathrm{GaAs}}+\propto_{\mathrm{GaInNA}}\right)\left(T_{s}-T_{c}\right) \\
(\Delta V)_{4} \text { junctions }=\propto_{\mathrm{GaInP}+\mathrm{GaAs}+\mathrm{GaInNA}+\mathrm{Ge}}\left(T_{s}-T_{c}\right)=\left(\propto_{\mathrm{GaInP}}+\propto_{\mathrm{GaAs}}+\propto_{\mathrm{GaInNA}}+\propto_{G e}\right)\left(T_{s}-T_{c}\right)
\end{array}
$$

According to such measurement results; we may write the following equations in general for any number of junctions: 


$$
\begin{gathered}
\propto_{\mathrm{GaInP}+\mathrm{GaAs}+\mathrm{GaInNA}} \equiv \propto_{\mathrm{GaInP}}+\propto_{\mathrm{GaAs}}+\propto_{\mathrm{GaInNA}} \\
\propto_{\mathrm{GaInP}+\mathrm{GaAs}+\mathrm{GaInNA}+\mathrm{Ge}} \equiv \propto_{\mathrm{GaInP}}+\propto_{\mathrm{GaAs}}+\propto \mathrm{G}_{\mathrm{aInNA}}+\propto_{\mathrm{Ge}}
\end{gathered}
$$

Such relations are found according to considering the whole MJPV cell receives heat from the same source and subjected to the environment of the same temperatures. So, its layers detect the same solar temperature $T_{h}=6100 \mathrm{~K}$, and the same surrounding of temperature: $T_{l}=298 \mathrm{~K}$. According to the previous analysis of the single junction solar cell, the incident radiation will gain an electric potential when crossing each junction which is proportional to the same thermal potential " $T_{h}-T_{l}$." Then, it is applied the previously found conclusions that considers the conversion of the thermal potential to electric potential is mainly actuated by the difference in the energy bandgaps, or Seebeck effect, at each junction according equations (23) and (24).

\section{Conclusions}

In this paper, we succeeded in introducing advanced physics of thermopiles, thermoelectric generators and multijunctions photovoltaic cells that explain how the potential of their output current is found as accumulation of the gained electric potentials during crossing their junctions. Such success highlights the nature of flow of electric current as flow of electromagnetic waves of electric potential. By involving the relation found by Goldsmid and Sharp between Seebeck coefficient and the energy bandgap, it was possible also to prove the similarity of the Photoelectric effect and the Seebeck effect. So, the thermopiles, the TEG, and the MJPV cells are defined in general, according to such advanced physics, as devices that convert the incident radiation into electric current of accumulated electric potentials during crossing the junctions of these devices due to the difference in the Seebeck effect of the materials of these junctions. According to such physics, it was possible also to prove the efficiencies of the photovoltaic cells may exceed the limit found by Shockley and Queisser as derived by classical physics of electric current and PV cells. Such advanced physics solves many conflicts in understanding the generation of electric potential in neural cells as will be introduced in future articles Insha' Allah.

\section{References}

[1] Abdelhady, S., A fundamental equation of thermodynamics that embraces electrical and magnetic potentials. J. Electromagnetic Analysis \& Applications 2010; 2: 162.

[2] Abdelhady, S., Cheng, C. H., Advanced Thermodynamics Engineering 1 ${ }^{\text {st }}$ Edn, (Scitus Academics. NY; 2018, p. 244.

[3] Abdelhady, S., An advanced review of thermodynamics of electromagnetism. International Journal of Research studies in Science, Engineering and Technology 2015; 3: 10.

[4] Abdelhady, S., An entropy approach to Tesla's discovery of wireless power transmission. J. Electromagnetic Analysis \& Applications 2013; 5:157.
[5] Abdelhady, S., Review of thermodynamics of systems that embraces the transfer of electric and magnetic energies, Journal of Physical Science and Application 2018; 8: 1 .

[6] Abdelhady, S., Innovative understanding of the duality confusion, the photovoltaic and magnetocaloric effects. Ain Shams Engineering Journal, Engineering Physics and Mathematics 2017; 8 In Press. https://doi.org/10.1016/j.asej.2017.03.006.

[7] Ryan, DT., Towards a cognitive-historical understanding of Michael Faraday's research: editor's introduction, Prospect Sci. 2006; 4: 1

[8] Tesla, N., The Effect of Static on Wireless Transmission, Electrical Experimenter, 1919; 1: 627.

[9] Christopher, R. H., Marshall, T. C., Stolzenburg, M., Estimations of charge transferred and energy released by lightning flashes," J. of geophysical research 2009; 114: 14203.

[10] Shockley, W., Queisser, H. J. Detailed balance limit of efficiency of p-n junction solar cells, Journal of Applied Physics 1961; 32: 510 .

[11] Goldsmid, H. J., Sharp, J. W., Estimation of thermal bandgap from the Seebeck coefficient measurements, Journal of electronic materials, Vol. 28, No. 7, (1999).

[12] Herwaarden, A. W. Sarro, Van, P. M. Thermal Sensors Based on The Seebeck Effect, Sensors and Actuators, 10, 321 (1986).

[13] Rowe, D. M. Thermoelectrics Handbook: Macro to Nano, 1st edn, Taylor \& Francis, NY 2006, p. 412.

[14] Bulusu, A., Walker, D. G., Review of electronic transport models for thermoelectric materials. Superlattices and Microstructures 2008; 44: 1 .

[15] Riffat, S. B., Ma, X., Thermoelectrics: A review of present and potential applications, Appl. Therm. Eng. 200; 3: 913.

[16] Skrabek, E. A., Trimmer, D. M. CRC Handbook of Thermoelectrics. $1^{\text {st }}$ edn, CRC Press, Boca Raton 1994, p. 129.

[17] B. J., A precise measurement of temperature difference using thermopiles, Experimental Thermal and Fluid Science 1990; 3: 265 .

[18] Snyder, G., Thermoelectric Efficiency and Compatibility, Physical Review Letters 2003; 91: 14.

[19] Smith, J. P., diSessa, A. A., Roschelle, J. Misconceptions reconceived: A constructivist analysis of knowledge in transition. Journal of the Learning Sciences, (1994); 3: 2.

[20] Prashantha, K., Wango, S., Smart power generation from waste heat by thermoelectric generator, International Journal of Mechanical and Production Engineering, 2016; Special Issue: 2020 .

[21] Sun, S., Qiu, K., Wu, Z., Xing, H. Y., Evaluation on HighEfficiency Thermoelectric Generation Systems Based on Differential Power Processing, IEEE Transactions on Industrial Electronics 2018; 65: 699. 
[22] Bell, L. E., "High Power Density Thermoelectric Systems", Proceedings of the $23^{\text {rd }}$ International Conference on Thermoelectrics, Adelaide, Australia, 2004: 210.

[23] Harman, T. C., et al. "Quantum Dot Superlattice Thermoelectric Materials and Devices", Science 2002; 297: 2229.

[24] Luque, A. Handbook of Photovoltaics Science and Engineering, 2nd edn Wiley, NY, 2010. p. 447.

[25] Tsakalakos L. Introduction to Photovoltaic Physics, Applications, and Technologies (CRC Press, Hoboken, New Jersey, 2010. p. 435.

[26] Abdelhady, S., Comments on Einstein's explanation of electrons, photons, and the photo-electric effect. Applied Physics Research 2011; 3:

230.http://dx.doi.org./5539/apr.v3n2p230/

[27] Burnett, B., The Basic Physics and Design of III-V Multijunction Solar Cells, NREL's III-V research group, 2002. p. 1283

[28] Friedman, D. J., Kurtz, S. R., Breakeven Criteria for the GaInNAs Junction in $\mathrm{GaInP} / \mathrm{GaAs} / \mathrm{GaInNAs} / \mathrm{Ge}$ Fourjunction Solar Cells, Prog. Photovolt: Res. Appl 2002; 10: 331 .

[29] Tanabe, K., Ehrenreich, H., Martin, J. H., Solar Photovoltaic Energy, Physics Today 1979; 32: 25.

[30] Herwaarden, A. V. The Seebeck Effect in Silicon ICs. Sensors and Actuators 1984; 6: 245. DOI 10.1016/02506874(84)85020-9

[31] Abdelhady, S, An entropy approach to a practical limit of the efficiencies of developed multijunction solar cells. J. Electromagnetic Analysis \& Applications6, 383-390, (2014).

[32] Abdelhady, S., Thermodynamic Analysis of Energy Flow in Optical Fiber Communication Systems. Applied Physics Research 2012; 4: 22. doi:10.5539/apr.v4n3p22

[33] Nolas, G. S, Sharp, J., Goldsmid, J., Thermoelectrics: Basic Principles and New Materials Developments, Springer; Amazon, Springer, 2001, p. 432.

[34] Zhang, Y., et al, Measurement of Seebeck coefficient perpendicular to SiGe superlattice layers, $21 \mathrm{st}$
International Conference on Thermoelectrics, Long Beach CA 2002, p. 26.

[35] Abdelhady, S. et al. Thermodynamics: Fundamentals and its Application in Science, Auris Reference, London, 2017, p. 142.

[36] Buljan, M., Mendes, J., Benitez, P., Minano, J. C., Recent trends in concentrated photovoltaics concentrators' architecture, J. Photonics Energy2014; 4: 1.

[37] Ian, M., et al, Performance of III-V Solar Cells as Indoor Light Energy Harvesters, IEEE Journal of Photovoltaics 2015: 2156.

[38] Simon Welzmiller et al, Increasing Seebeck Coefficients and Thermoelectric Performance of $\mathrm{Sn} / \mathrm{Sb} / \mathrm{Te}$ and $\mathrm{Ge} / \mathrm{Sb} / \mathrm{Te}$ Materials by Cd Doping; Adv. Electron. Mater. 2012; 1: 1500266 .

[39] Stephanie Essig, Myles A. Steiner, Christopher, Realization of GaInP/Si Dual-Junction Solar Cells With 29.8\% 1-Sun Efficiency Published in: IEEE Journal of Photovoltaics 2016; 6: 4 .

[40] Arto, A., Tukiainen, A., Polojärvi, V., Mircea, Guina. Performance assessment of multijunction solar cells incorporating GaInNAsSb, Nanoscale Research Letters 2014; 9:61.

\section{Biography}

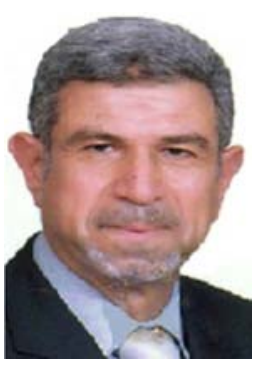

Salama Abdelhady is a full Professor of energy systems in Aswan University, has obtained his $\mathrm{Ph}$. D. degree from Brn University in 1975 , He was the co-Founder \& Dean of the Faculty of energy engineering in Aswan University, he has many researches in the field of thermodynamics, heat transfer, energy conservation and renewable energy, led many projects in the field of reverse engineering and CSP, he is a member in the supreme committee of power engineering for three periods, he was a visiting professor in Lehigh university, he was the head of departments of power engineering in MTC, High Institute of Energy, CIC, and Heliopolis. 